\title{
COMMUTATIVITY RESULTS FOR SEMIPRIME RINGS WITH DERIVATIONS
}

\author{
MOHAMAD NAGY DAIF \\ Department of Mathematics \\ Faculty of Science \\ Al-Azhar University \\ Nasr City 11884 \\ Cairo, EGYPT
}

(Received March 8, 1996 and in revised form October 10, 1996)

\begin{abstract}
We extend a result of Herstein concerning a derivation $d$ on a prime ring $R$ satisfying $[d(x), d(y)]=0$ for all $x, y \in R$, to the case of semiprime rings. An extension of this result is proved for a two-sided ideal but is shown to be not true for a one-sided ideal. Some of our recent results dealing with $\mathrm{U}^{*}$ - and $\mathrm{U}^{* *}$ - derivations on a prime ring are extended to semiprime rings. Finally, we obtain a result on semiprime rings for which $d(x y)=d(y x)$ for all $x, y$ in some ideal $U$.
\end{abstract}

KEY WORDS AND PHRASES: Semiprime ring, derivation, commutator, and central ideal. 1991 AMS SUBJECT CLASSIFICATION CODES: 16W25, 16U80, 16 N60.

\section{INTRODUCTION}

In his note on derivations, Herstein [1] showed that if a prime ring $\mathbf{R}$ of characteristic not 2 admits a nonzero derivation $d$ such that $[d(x), d(y)]=0$ for all $x, y$ in $R$, then $R$ is commutative. Here, we give an easy but elegant extension of this result in the case when $\mathbf{R}$ is semiprime. Moreover, by making use of a more recent result of Bell and Martindale [2], we can get a more general theorem for a semiprime ring, which requires the condition $[d(x), d(y)]=0$ to hold only on some ideal of $R$. We notice that a one-sided ideal would not work in this new theorem, the example given by Bell and Daif [3] is a counter-example.

Recently, Bell and Daif [3] introduced the notions of $U^{*_{-}}$and $U^{*_{*}}$ - derivations $d$ on a prime ring $R$, where $U$ is a nonzero right ideal of $R$. If $d$ is a derivation on $R$ such that $d(x) d(y)+d(x y)=$ $d(y) d(x)+d(y x)$ for all $x, y \in U$, we say that $d$ is a $U^{*}$ - derivation; and if $d(x) d(y)+d(y x)=d(y) d(x)$ $+d(x y)$ for all $x, y \in U$, we call $d a U^{\star \star}$ - derivation. We proved that if $d$ is a nonzero $U^{\star_{-}}$or $U^{\star_{*}}$ derivation, then either $R$ is commutative or $d^{2}(U)=d(U) d(U)=\{0\}$. This result yielded a result of Bell and Kappe [4]. We also studied derivations $d$ satisfying $d(x y)=d(y x)$ for all $x, y \in U$. For formal reasons, we call d a $\mathrm{U}^{\star \star \star}$ - derivation if it satisfies this condition. In this note, we extend these results to the semiprime case. We will show for a nonzero $U^{\star}$ - or $U^{\star \star}$ - derivation $d$ that $d(U)$ centralizes $[U, U]$. In the event that $U$ is a two-sided ideal, we show that $R$ contains a nonzero central ideal. The same conclusion is obtained when $R$ admits $a U^{\star \star \star *}$ - derivation which is nonzero on $U_{\text {. }}$

For the ring $R, Z$ will denote the center of $R$. For elements $x, y \in R$, the commutator $x y-y x$ will be written as $[x, y]$; and for a subset $U$ of $R$, the set of all commutators of elements of $U$ will be written as $[U, U]$. We will make extensive use of the familiar commutator identities $[x, y z]=y[x, z]+$ $[x, y] z$ and $[x y, z]=x[y, z]+[x, z] y$. 
To achieve our purposes, we mention the following results.

(A) [1, Theorem 1] Let $R$ be any ring and $d$ a derivation of $R$ such that $d^{3} \neq 0$. Then the subring of $R$ generated by all $d(r), r \in R$, contains a nonzero ideal of $R$.

(B) [2, Theorem 3] Let $R$ be a semiprime ring and $U$ a nonzero left ideal. If $R$ admits a derivation $d$ which is nonzero on $U$ and centralizing on $U$, then $R$ contains a nonzero central ideal.

(C) [5, Lemma 1] Let $R$ be a semiprime ring and $U$ a nonzero two-sided ideal of $R$. If $x \in R$ and $x$ centralizes $[U, U]$, then $x$ centralizes $U$.

\section{EXTENSIONS OF HERSTEIN'S THEOREM}

THEOREM 2.1. Let $R$ be a semiprime ring and $d$ a derivation of $R$ with $d^{3} \neq 0$. If $[d(x), d(y)]=0$ for all $x, y \in R$, then $R$ contains a nonzero central ideal.

PROOF. By (A), the subring generated by $d(R)$ contains a nonzero ideal $U$ of $R$. By our hypothesis, $U$ is commutative; hence $U^{2} \subseteq Z$. But $R$ is semiprime, hence $U \neq\{0\}$ implies $U^{2} \neq\{0\}$, which completes the proof.

Now we aim to extend the theorem of Herstein in the situation when the ring is semiprime and the condition $[d(x), d(y)]=0$ is merely satisfied on an ideal of the ring.

THEOREM 2.2. Let $R$ be a two-torsion-free semiprime ring and $U$ a nonzero two-sided ideal of $R$. If $R$ admits a derivation $d$ which is nonzero on $U$ and $[d(x), d(y)]=0$ for all $x, y \in U$, then $R$ contains a nonzero central ideal.

PROOF. We are given that

$$
[d(x), d(y)]=0 \text { for all } x, y \in U .
$$

Replacing y by yz, we therefore obtain

$$
d(y)[d(x), z]+[d(x), y] d(z)=0 \text { for all } x, y, z \in U .
$$

Putting $z=z r$ where $z \in U$ and $r \in R$, we now get

$$
d(y) z[d(x), r]+[d(x), y] z d(r)=0 \text { for all } x, y, z \in U, r \in R \text {. }
$$

Now substitute $r=d(t), t \in U$, to get

$$
[d(x), y] z d^{2}(t)=0 \text { for all } x, y, z, t \in U .
$$

Let $\left\{P_{\alpha}: \alpha \in \Lambda\right\}$ be a family of prime ideals of $R$ such that $\bigcap_{\alpha} P_{\alpha}=\{0\}$. Now (2.4) yields $[d(x), y] x R d^{2}(t)=\{0\}$ for all $x, y, z, t \in U$; hence for each $P_{\alpha}$, we either have

(a) $[d(x), y] U \subseteq P_{\alpha}$ for all $x, y \in U$,

or

(b) $d^{2}(U) \subseteq P_{\alpha}$.

Call $P_{\alpha}$ an (a)-prime ideal or (b)-prime ideal according to which of these conditions is satisfied.

Note that $[d(x), y] R U \subseteq P_{\alpha}$ for each (a)-prime $P_{\alpha}$, so either $[d(x), y] \in P_{\alpha}$ for all $x, y \in U$ or $\mathbf{U} \subseteq \mathbf{P}_{\alpha}$. In either event,

$$
[d(x), y] \in P_{\alpha} \text { for all } x, y \in U \text { and all (a)-prime } P_{\alpha} \text {. }
$$

Now consider (b)-prime ideals. Taking $x, y \in U$, we have $d^{2}(x y)=d^{2}(x) y+x d^{2}(y)+$ $2 d(x) d(y) \in P_{\alpha}$, so $2 d(x) d(y) \in P_{\alpha}$ for all $x, y \in U$. Replacing $y$ by zy shows that 


$$
\operatorname{2d}(x) z d(y) \in P_{\alpha} \text { for all } x, y, z \in U \text {; }
$$

hence

$$
2 d(x) \operatorname{Rzd}(y) \subseteq \mathbf{P}_{\alpha} \text { and } 2 \mathrm{~d}(\mathrm{x}) \mathrm{zRd}(\mathrm{y}) \subseteq \mathbf{P}_{\alpha} \text { for all } \mathbf{x}, \mathbf{y}, \mathbf{z} \in \mathbf{U}
$$

It follows that either $d(U) \subseteq P_{\alpha}$, or $2 d(x) y$ and $2 y d(x) \in P_{\alpha}$ for all $x, y \in U$. In either case,

$$
2[d(x), y] \in P_{\alpha} \text { for all } x, y \in U \text { and (b)-prime } P_{\alpha} \text {. }
$$

Thus, for all $x, y \in U$ we have (by (2.5) and (2.8)) that $2[d(x), y] \in \bigcap_{\alpha} P_{\alpha}=\{0\}$; and since $R$ is 2-torsionfree, $[d(x), y]=0$ for all $x, y \in U$. In particular, $[d(x), x]=0$ for all $x \in U$, so the theorem follows by (B).

REMARK. We notice that Theorem 2.2 is not true in the case when $U$ is one-sided. Let $R$ be the ring of all $2 \times 2$ matrices over a field $F$; let $U=\left[\begin{array}{l}10 \\ 00\end{array}\right] R$. Let $d$ be the inner derivation given by $d(x)=x\left[\begin{array}{l}01 \\ 0\end{array}\right]-\left[\begin{array}{l}01 \\ 0\end{array}\right] x$ for all $x \in R$. For any two elements $x$ and $y$ in $U$, we have that $[d(x), d(y)]=0$, but the conclusion of the theorem is not true.

\section{EXTENDING RESULTS ON U*-AND U**- DERIVATIONS}

THEOREM 3.1. Let $R$ be a semiprime ring and $U$ a nonzero right ideal of $R$. If $R$ admits a nonzero $U^{*}$-derivation $d$, then $d(U)$ centralizes [U,U].

PROOF. The condition that $d$ is a $U^{*}$ - derivation yields

$$
[d(x), d(y)]=[d(y), x]+[y, d(x)] \text { for all } x, y \in U .
$$

Proceeding exactly as in [3], we see that

$$
[d(x), x] U R\left(d(x)+d^{2}(x)\right)=\{0\} \text { for all } x \in U \text {. }
$$

Since $\mathbf{R}$ is semiprime, it must have a family $\left\{P_{\alpha}: \alpha \in \Lambda\right\}$ of prime ideals such that $\bigcap_{\alpha} P_{\alpha}=\{0\}$. Let $P$ be a typical one of these. By (3.2) we see that for each $x \in U$, either $[d(x), x] U \subseteq P$ or $d(x)+d^{2}(x)$ $\in P$. We now use the kind of argument employed in the proof of Theorem 2.2, in effect performing the calculations of [3] modulo $P$; we arrive at the conclusion that

$$
\text { either } \mathbf{d}(\mathbf{U}) \mathbf{U} \subseteq \mathbf{P} \text { or }[\mathbf{x}+\mathbf{d}(\mathbf{x}), \mathbf{R}] \subseteq \mathbf{P} \text { for all } \mathbf{x} \in \mathbf{U}
$$

In the first case, we can again employ the argument of [3] modulo $P$, obtaining the result that

$$
\text { either } U \subseteq P \text { or }[d(x), d(t)] \in P \text { for all } x, t \in U \text {. }
$$

Returning to the second possibility in (3.3), we assume that $[x+d(x), R] \subseteq P$. We then have $[x, d(t)]+[d(x), d(t)] \in P$ for all $x, t \in U$. But from $(3.1)$ we have $[d(x), d(t)]+[x, d(t)]=[t, d(x)]$, hence we have

$$
[t, d(x)] \in P \text { for all } x, t \in U \text {. }
$$

Putting $t=\operatorname{td}(y)$ and using (3.5), we get

$$
t[d(y), d(x)] \in P \text { for all } x, y, t \in U \text {. }
$$

From (3.6) we have $U R[d(y), d(x)] \subseteq P$ for all $x, y \in U$. Consequently, either $U \subseteq P$ or $[d(x), d(t)] \in P$ for all $x, t \in U$, which are the same alternatives as in (3.4). 
If we consider the case $U \subseteq P$, then from (3.1) we get $[d(x), d(t)] \in P$ for all $x, t \in U$. Therefore, we always have $[d(x), d(t)] \in P$ for all $x, t \in U$. Now using the fact that $\bigcap_{\alpha} P_{\alpha}=\{0\}$, we conclude that $[d(x), d(t)]=0$ for all $x, t \in U$. From our hypothesis, we have $d(x t)=d(t x)$ for all $x, t \in U$. This means that $d([x, t])=0$ for all $x, t \in U$. But $d([x, t] z)=d(z[x, t])$, hence $[x, t] d(z)=d(z)[x, t]$ for all $x, z, t \in U$. Thus $d(U)$ centralizes $[U, U]$ as required.

Similar conclusions as in the proof of Theorem 3.1 lead us to the same conclusion in the case that $d$ is a $U^{* *}$ - derivation. Therefore, we have

THEOREM 3.2. Let $R$ be a semiprime ring and $U$ a nonzero right ideal of $R$. If $R$ admits a nonzero $U^{* *}$ - derivation, then $d(U)$ centralizes $[U, U]$.

COROLLARY. Let $R$ be a semiprime ring and $U$ a nonzero two-sided ideal of $R$. If $R$ admits a $U^{*_{-}}$or $U^{*_{*}}$ - derivation d which is nonzero on $U$, then $R$ contains a nonzero central ideal.

PROOF. By Theorems 3.1 and 3.2, d(U) centralizes [U,U]. By (C), we get that d(U) centralizes $U$. The result now follows by (B).

THEOREM 3.3. Let $R$ be a semiprime ring and $U$ a nonzero two-sided ideal of $R$. If $R$ admits a $U^{\star \star \star}$ - derivation $d$ which is nonzero on $U$, then $R$ contains a nonzero central ideal.

PROOF. Since $d(x y)=d(y x)$ for all $x, y \in U$, the argument at the end of the proof of Theorem 3.1 shows that $d(U)$ centralizes $[U, U]$. The result now follows as in the proof of the Corollary.

ACKNOWLEDGEMENT. I am truly indebted to Prof. Howard E. Bell for his sincere suggestions and great help which made the paper in its present form.

\section{REFERENCES}

[1] HERSTEIN, I. N., “A note on derivations,” Canad. Math. Bull. 21(1978), 369-370.

[2] BELL, H. E. and MARTINDALE, W. S. III, "Centralizing mappings of semiprime rings," Canad. Math. Bull. 30(1987), 92-101.

[3] BELL, H. E. and DAIF, M. N., “ On derivations and commutativity in prime rings," Acta Math. Hungar. 66(4)(1995), 337-343.

[4] BELL, H. E. and KAPPE, L. C., “ Rings in which derivations satisfy certain algebraic conditions," Acta Math. Hungar. 53(1989), 339-346.

[5] DAIF, M. N. and BELL, H. E., "Remarks on derivations on semiprime rings," Internat. J. Math. \& Math. Sci. 15(1992), 205-206. 


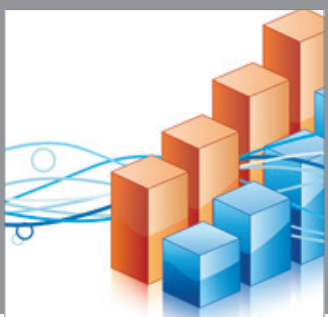

Advances in

Operations Research

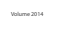

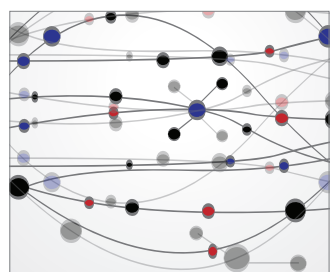

\section{The Scientific} World Journal
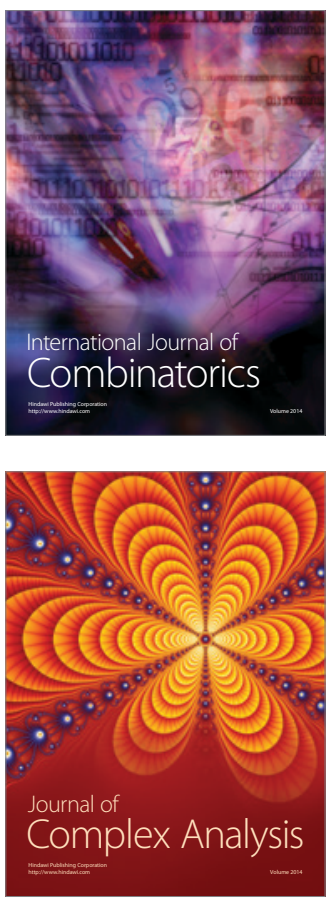

International Journal of

Mathematics and

Mathematical

Sciences
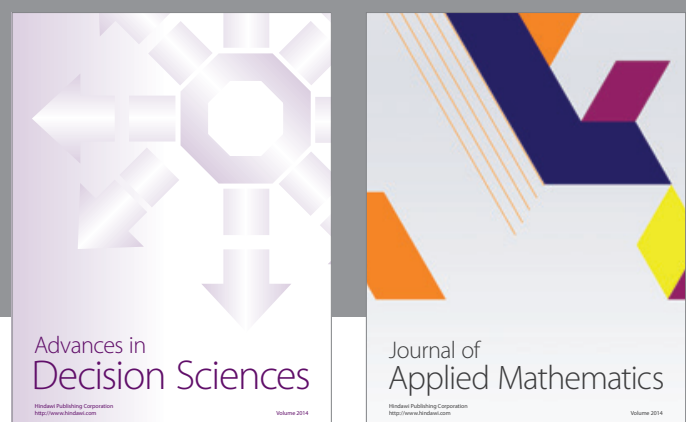

Journal of

Applied Mathematics
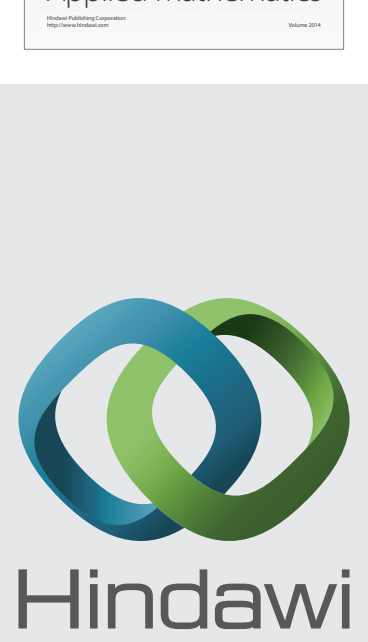

Submit your manuscripts at http://www.hindawi.com
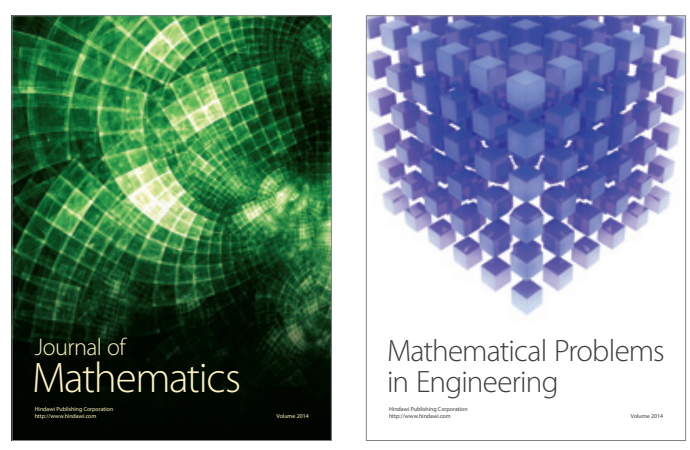

Mathematical Problems in Engineering
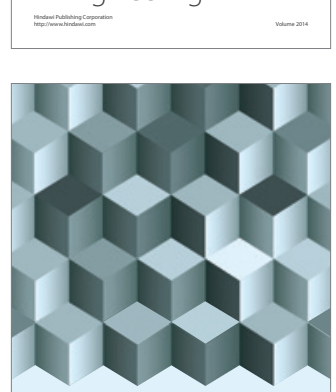

Journal of

Function Spaces
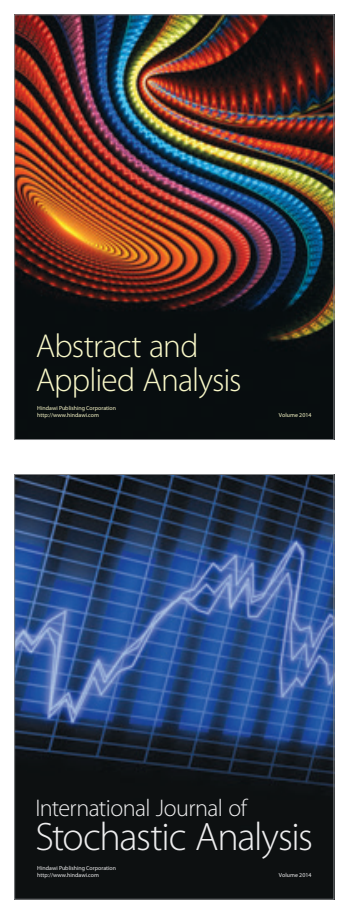

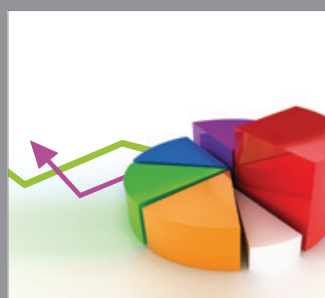

ournal of

Probability and Statistics

Promensencen
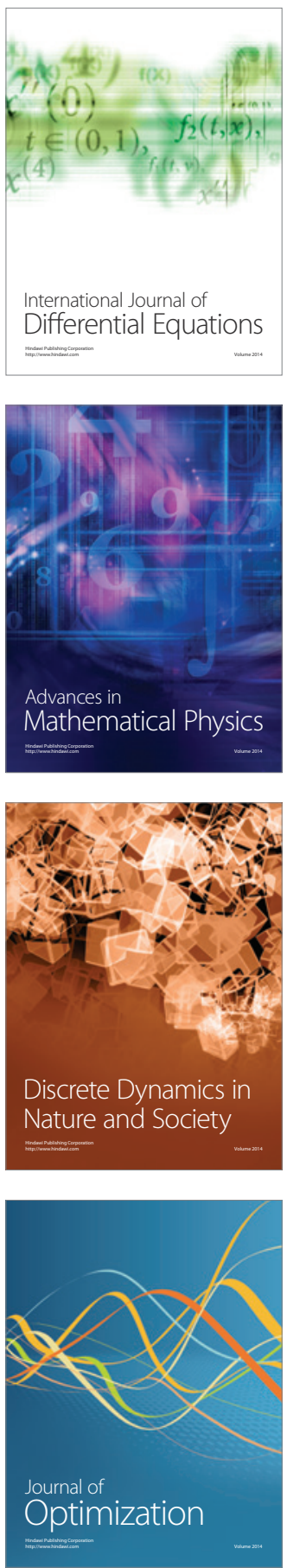